\title{
Evaluating the Performance of Virtual Reality Navigation Techniques for Large Environments
}

\author{
Kurtis Danyluk ${ }^{1}$ and Wesley Willett ${ }^{1}$ \\ University of Calgary, Calgary, AB, Canada \\ ktdanylu, wesley.willett@ucalgary.ca
}

\begin{abstract}
We present results from two studies comparing the performance of four different navigation techniques (flight, teleportation, worldin-miniature, and 3D cone-drag) and their combinations in large virtual reality map environments. While prior work has individually examined each of these techniques in other settings, our study presents the first direct comparison between them in large open environments, as well as one of the first comparisons in the context of current-generation virtual reality hardware. Our first study compared common techniques (flight, teleportation, and world-in-miniature) for search and navigation tasks. A follow-up study compared these techniques against 3D cone drag, a direct-manipulation navigation technique used in contemporary tools like Google Earth VR. Our results show the strength of flight as a stand-alone navigation technique, but also highlight five specific ways in which viewers can combine teleportation, world-in-miniature, and 3D cone drag with flight, drawing on the relative strengths of each technique to compensate for the weaknesses of others.
\end{abstract}

Keywords: Human computer interaction (HCI) - Virtual reality · Digital Maps · Navigation

\section{INTRODUCTION}

Recent advances in consumer-grade immersive virtual reality (VR) technology have made it increasingly easy to create large, open, and detailed virtual environments. Many fields already use these systems to accurately portray information that is challenging to present on traditional 2D flat displays. For example, urban informatics, geology, and infrastructure planning all benefit from the detailed and flexible perspectives provided by immersive virtual reality.

However, existing work has rarely compared navigation techniques for large spaces, instead focusing on novel navigation techniques like GiAnt [2] and cubemaps [12]. Those evaluations that do exist [6] consider a limited set of techniques [9] or focus on small virtual worlds [3]. Moreover, contemporary navigation techniques like 3D cone drag [8], which have been designed for large environments, remain largely unexamined in the research literature.

We present a direct comparison of four navigation techniques - flight, teleportation, world-in-miniature, and 3D cone drag (Figure 1) - in large immersive VR environments and discuss how they can be combined. To compare 
these approaches, we developed a test-bed that supports all four techniques and conducted studies in which participants performed navigation and search tasks within a virtual mountainous environment. We examined when each technique performed best, which techniques viewers preferred to use, and how their navigation behavior changed when using each technique, especially when they had access to several. Based on our evaluations, we contribute a comparison of flight, teleportation, world-in-miniature, and 3D cone drag that showcases the all-around effectiveness of flight, as well as the strengths and weaknesses of the other techniques. We also examine five specific ways in which participants combined these navigation techniques to overcome their individual weaknesses.

\section{RELATED WORK}

Our work builds on a variety of prior examinations of interactive navigation in virtual reality environments, including early work on VR camera control, as well as more recent techniques for navigating in large environments. Ware and Osborne [18] introduced and evaluated several navigation metaphors, including techniques they referred to as flying vehicle control and scene-in-hand. Due to the limitations of these early systems, scenes were limited to single objects and camera orientation was entirely controlled as part of the navigation technique, rather than by the viewer's head motion. Later work, including Stoakley et al.'s "Virtual reality on a WIM" [16] introduced worlds-in-miniature, which allowed viewers to navigate by manipulating a token placed in a miniature recreation of the scene. Much like earlier work, Stoakley et al.'s system still relied on token orientation rather than the viewer's head position to provide camera orientation.

In contrast, most contemporary VR systems use the viewer's head position to provide camera orientation, and rely on navigation techniques like flight or teleportation to change the viewer's position in the scene. Additionally, contemporary systems also use techniques like automatic scaling [2] or cubemaps [17] [12] to adjust navigation speed and reduce disorientation. Techniques like 3D cone drag [8] also extend the direct-manipulation metaphor of scene-in-hand to modern VR systems, allowing viewers to grab, manipulate, and move the scene.

Physical locomotion for navigation in VR is another popular navigation method, with many implementations of walking $[15,19]$. However, because of the large scale of map-like environments, most simple realizations of locomotion in VR are inadequate in large scenes. More complex imlplementations, such as redirected walking [14] or redirected teleportation [11], allow locomotion to function within large virtual spaces, but do not reduce the physical effort required to walk across a potentially kilometers-long scene. Scaling alternatives such as GulliVR [10] offer a solution to this problem, but drastically change the viewers' perspective of a scene. Other locomotion alternatives, such as virtual surfboards [7] or finger-walking [19] use locomotion as a controller for another navigation technique, normally flight.

Early comparisons of basic flight and teleportation in relatively small environments show that teleportation can be faster than flight but causes greater 


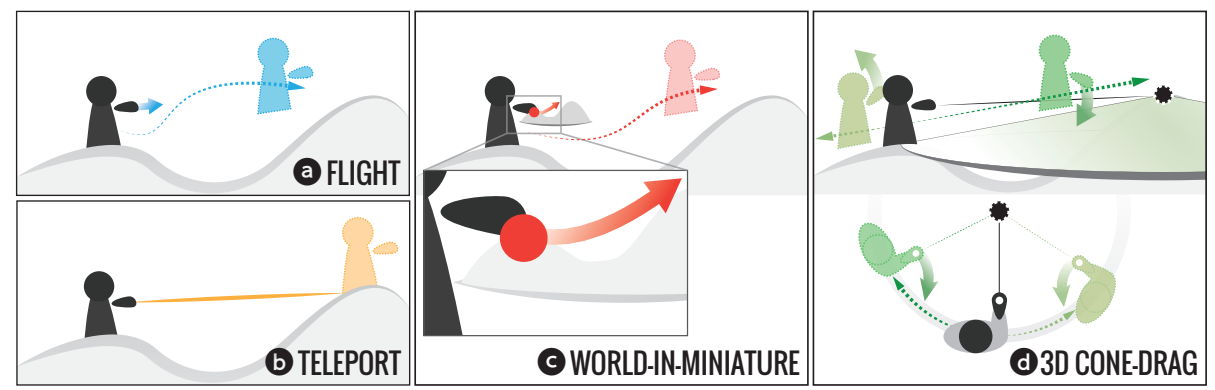

Fig. 1. The four different VR navigation techniques:(a) flight, (b) teleport, (c) worldin-miniature, (d) 3D cone drag.

disorientation [4]. Moreover, a large body of work suggests that constant velocity flight is less disorienting than accelerated flight, regardless of velocity [4]. However, there exists little work that directly compares techniques other then flight and teleportation [6].

\section{NAVIGATION IN LARGE VR SPACES}

Large VR spaces are a challenge because their sheer size rules out navigation techniques like locomotion, which most closely resemble navigation in the physical world. In large spaces, origins and destinations may be far apart, and viewers need mechanisms for transitioning these distances quickly yet precisely. Here, we introduce four common yet contrasting techniques we chose to compare in our studies.

Flight is one of the earliest navigation techniques for VR [18]. When using this technique, a viewer moves through virtual environments as if they were controlling a flying vehicle, like a helicopter (Figure 1a). Flight involves a smooth, predictable, continuous, and reversible translation.

World-in-miniature allows the viewer to

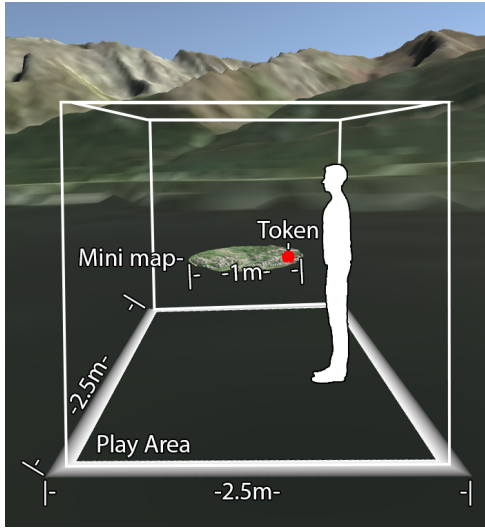

Fig. 2. The $2.5 \mathrm{~m}$ play area and $1 \mathrm{~m}$ diameter mini-map configuration used in our test environment. change their position by manipulating a version of themselves in a miniature version of the scene (Figure 1c). We use a $1 \mathrm{~m}$ diameter mini-map, located at the center of the viewer's play area (Figure 2).

3D Cone Drag allows the viewer to directly manipulate the scene as though they were directly grabbing and dragging the landscape. We examine 3D Cone Drag because of its prominent use in Google Earth VR [8], a popular commercial application which involves exactly the type of large scene navigation tasks that we are interested in exploring. 


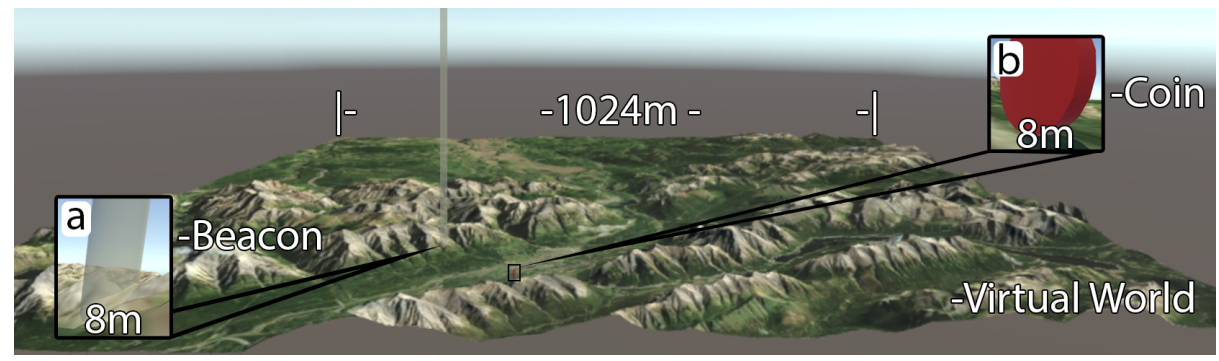

Fig. 3. Overview of the entire virtual world used during the study tasks. Insets show beacons (a) used for navigation tasks and coins (b) used for search tasks

Teleportation allows the viewer to specify a point in space and then directly and instantly move to it (Figure $1 \mathrm{~b}$ ). The most obvious advantage of teleportation is that transit is instant. Further, because there are no intermediate transitions, users do not experience any velocity in navigation, avoiding a common cause of cybersickness.

Of these techniques, world-in-miniature requires an additional navigation aid - in this case, a mini-map showing a small-scale, version of the surrounding environment. However, mini-maps also provide an alternative perspective that can help viewers evaluate distances and see terrain outside of their immediate field of view. In particular, mini-maps may be a useful navigation aid when using teleportation, where they make it possible for viewers to teleport to parts of the virtual world that are not directly visible from their current location.

\section{STUDIES}

We conducted a pair of studies that examine the trade-offs between these techniques and how viewers might combine them. In both studies, we compared (1) which techniques allowed viewers to navigate and search more quickly in large virtual environments and (2) how each technique impacted viewers' sense of orientation. We also characterized (3) viewers qualitative preferences for and experiences with these four techniques, and (4) examined how viewers combined the approaches to compensate for their strengths and weaknesses.

\subsection{Study Setup}

Test Environment We developed our study environment for the HTC Vive VR headset. The implementation used Unity 5.6 with $\mathrm{C \#}$ and relied on elevation data from Amazon Web Services and satellite imagery from Bing Maps. Using these we created large virtual worlds at scales between 1:78271 and 1:4.7. Our test map included a $124 \mathrm{~km} \times 124 \mathrm{~km}$ region centered around the hamlet of Exshaw, Alberta. This area is situated at the edge of the Canadian Rockies, near 
Banff National Park, and features a diverse mix of flat, rolling, and mountainous terrain. We rendered this region at a scale of roughly 1:120, resulting in a $1024 \mathrm{~m} \times 1024 \mathrm{~m}$ virtual terrain (Figure 3 ). Within this virtual world, the viewer occupied a $2.5 \mathrm{~m} \times 2.5 \mathrm{~m}$ play area (mirroring the size of the physical test area) within which they could move and interact freely (Figure 2).

\subsection{Tasks and Measures}

Tasks. We asked participants to perform two different kinds of common spatial wayfinding and orientation tasks [13]. These included navigation tasks which required viewers to move as quickly as they could to a known location, and search tasks which required them to explore the virtual world in order to locate a hidden object. These are similar to the tasks used by Darken and Peterson [13]. Each trial consisted of one task.

Navigation tasks involved moving from a starting position to a large, clearly visible beacon placed at a random location in the environment (Figure 3a). The beacons also appeared as bright yellow pins on the mini-map.

Search tasks involved finding and collecting a large red coin (Figure 3b) placed randomly in the environment. Coins were not marked on the mini-map.

Measures. During both studies we tracked participants' position in the virtual environment We also collected timing data for each task, along with detailed notes on participants' behaviors and vocalizations.

To gauge participants' spatial awareness, we asked them to complete a pointback test immediately after they finished each task. Upon reaching a beacon or coin, the interface prompted participants to point back towards the location of the previous coin or beacon. We measured point-back error by calculating the acute horizontal angle between where the viewers pointed and the actual position of the previous beacon/coin.

After the study, participants completed a 5-point Likert survey rating the difficulty and enjoyability of the navigation techniques. Finally, we asked participants to discuss their favorite and least favorite navigation techniques.

\subsection{Study 1: Flight, Teleportation, and World-in-Miniature}

Study 1 compared flight, teleportation, and world-in-miniature. We also evaluated the effect of a mini-map in the flight and teleportation conditions and examined participants' behavior when they could combine all of the techniques.

Participants and Procedure. We recruited 10 Participants (2 female / 8 male, ages 22-47). Their VR experience ranged between none (7), extensive gaming (2), and development experience (1). We refer to these participants using codes P1-P10.

Participants performed 5 repetitions of the

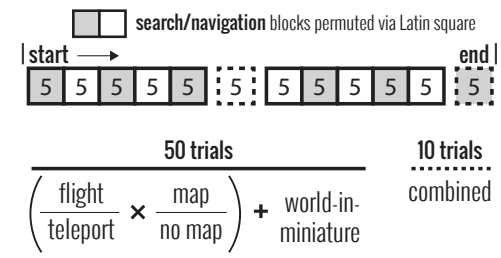
two task types in 6 different experimental conditions for a total of 60 trials. They alternated between 5 -trial blocks of search 
and navigation tasks. In blocks 1-5 and 7-11 participants alternated between 5 different experimental conditions - flight and teleportation (each with/without mini-map) plus world-in-miniature. Blocks 6 and 12 used a combined interface where participants had access to flight, teleportation, world-in-miniature, and the mini-map simultaneously. We permuted both task and condition using a Latin square design. Participants could rest between each block, and we asked them to remove the VR headset and take an enforced break after every three blocks. Participants also performed a 10-minute training block where the experimenter familiarized them with all the navigation techniques and how to perform each task. After all the tasks, participants debriefed with the experimenter and completed a follow-up questionnaire. This took roughly 1 hour to complete.

Quantitative Results. Due to growing concern in a variety of fields about the use of null hypothesis significance testing [5] we analyze our results using estimation techniques and report effect sizes with confidence intervals (CI) rather than p-value statistics. This reporting methodology is consistent with recent APA recommendations [1]. We first computed average scores for each participant, then computed averages and $95 \%$ confidence intervals using the aggregate scores, applying a Bonferroni correction to control for multiple comparisons. We also computed pairwise differences between conditions, again using 95\% confidence intervals with a Bonferroni correction. Our complete analysis as well as additional explanatory figures can be viewed as supplementary material. ${ }^{1}$

Mini-map. Although we included variations of flight and teleport both with and without a mini-map, the inclusion of a mini-map had little impact on task performance. For navigation tasks, pairwise comparisons (Figure 4) showed no differences in task duration or point-back error between the map and no-map variations for either flight or teleportation. For search tasks, we only observed one clear case in which the map appeared to impact performance. Participants took an average of $16.5 \mathrm{~s}(\mathrm{CI}=[14.3,18.9])$ to complete search tasks while using flight with no-map, compared to $21.8 \mathrm{~s}(\mathrm{CI}=[18.5,25.8])$ with a map. We saw no clear difference in point-back error across any of the variations, and no clear interactions between the mini-map and any of the interaction techniques. In light of this, we combined data from the map and no-map conditions in all subsequent analysis of Study 1.

Task Duration. Participants using flight took an average of $15.9 \mathrm{~s}$ ( CI $=$ $[12.1,24.6])$ to complete navigation trials and $19.1 \mathrm{~s}(\mathrm{CI}=[15.5,23.1])$ to complete search trials. World-in-miniature was slower, averaging 18.5s $(\mathrm{CI}=[13.0,28.1])$ for navigation trials and 37.0s $(\mathrm{CI}=[28.4,50.8])$ for search trials. Teleportation was also slower, averaging $21.1 \mathrm{~s}(\mathrm{CI}=[16.2,27.3])$ for navigation tasks and $56.9 \mathrm{~s}$ $(\mathrm{CI}=[40.6,84.3])$ for search tasks.

\footnotetext{
${ }^{1}$ https://www.dropbox.com/s/muyzsbge8zlwpi1/Submission\%20Package.zip?dl=0
} 


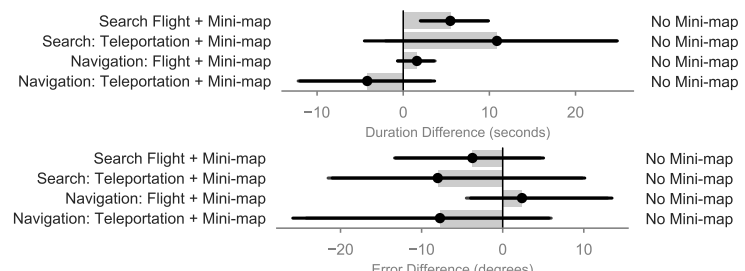

Fig. 4. Pairwise comparison of task duration (top) and point-back error (bottom) for flight and teleportation with and without a mini-map. Error Bars show 95\% CIs.
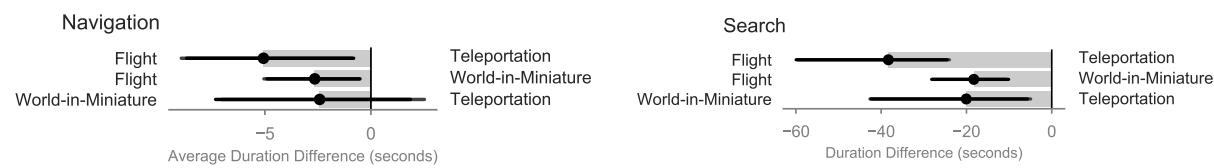

Fig. 5. Pairwise comparisons of task duration between conditions for navigation tasks (left) and search tasks (right) in Study 1. All error bars show 95\% CIs.

Pairwise comparisons (Figure 5) show that flight was faster than either teleportation or world-in-miniature for navigation tasks, but show no clear difference between teleportation and world-in-miniature. For search tasks, flight outperformed other approaches by a considerable margin, and world-in-miniature clearly outperformed teleportation. Although flight performed best, 3 of 10 participants noted that they felt world-in-miniature was as fast or faster than flight, even though they performed more slowly with it.

Orientation. Point-back error was comparable between flight and world-inminiature for both task types. In navigation trials the average point-back error for flight was $16.9^{\circ}(\mathrm{CI}=[10.0,30.8])$ while world-in-miniature averaged $21.1^{\circ}$ $(\mathrm{CI}=[14.7,27.9])$. For search trials flight averaged $17.7^{\circ}(\mathrm{CI}=[13.2,23.5])$ and world-in-miniature $21.7^{\circ}(\mathrm{CI}=[12.7,32.5])$. Teleportation introduced much more error, averaging $25.5^{\circ}(\mathrm{CI}=[17.4,38.0])$ in navigation trials and $38.2^{\circ}(\mathrm{CI}=$ $[27.5,48.7])$ in search. Pairwise comparisons (Figure 6) show similarity between flight and world-in-miniature and their divergence from teleportation.

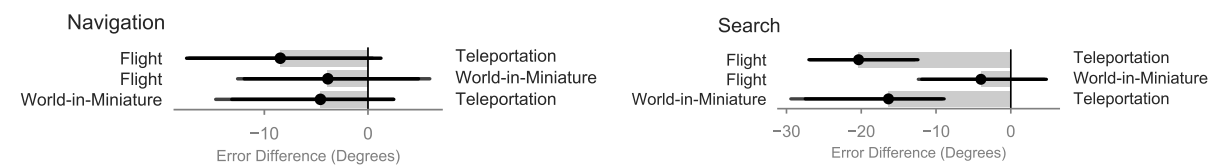

Fig. 6. Pairwise comparisons of point-back error between conditions for navigation tasks (left) and search tasks (right) in Study 1. All error bars show 95\% CIs. 


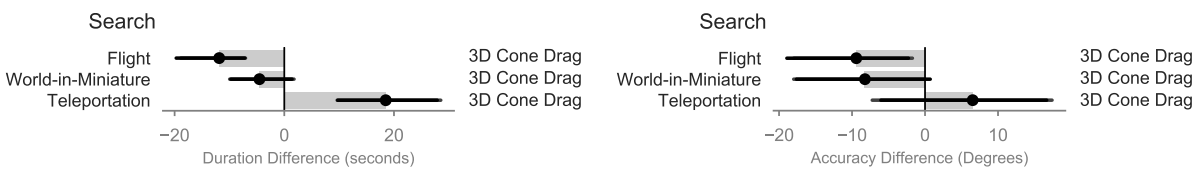

Fig. 7. Pairwise comparisons of task duration (left) and point-back error (right) for Study 2. Error bars show $95 \%$ CIs.

\subsection{Study 2: Comparison with 3D Cone Drag}

The second study compared the techniques from the first study (flight, teleportation, and world-in-miniature) with 3D cone drag, the navigation technique used by Google Earth VR [7].

Participants and Procedure. We recruited 8 participants (2 female / 6 male, ages 22-43), balancing four repeat participants (P6-P10) from Study 1, with four new participants (P11-14).

Because differences between the navigation techniques were more pronounced for search tasks, we did not include navigation tasks in Study 2. Instead, participants completed 50 search trials split across 10 blocks. To capture more data about the new condition while reducing participant fatigue, we biased the number of blocks allocated to 3D cone drag (4 blocks) and flight (2 blocks) and included only one block each of teleport and world-in-miniature to serve as training for the combined condition. We permuted block order using a Latin Square. To explore how participants combined all four techniques, we included two combined blocks at the end of the study.

As in Study 1, participants had the opportunity to rest between each block, and we asked them to remove the VR headset and take an enforced break after 4 blocks. We also used the same pre- and post-study procedures as in Study 1.

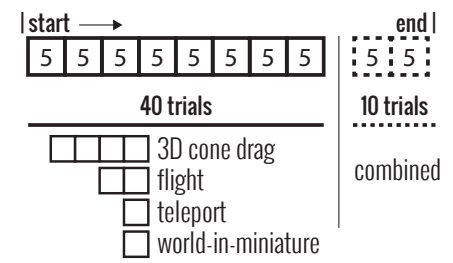

Task Duration. Participants performed the best

with flight, taking an average of $19.2 \mathrm{~s}(\mathrm{CI}=[16.3,23.5])$ to complete tasks. This was faster than world-in-miniature which took on average $26.5 \mathrm{~s}$ (CI $=$ $[22.6,31.9])$, 3D cone drag which took on average 31.0s $(\mathrm{CI}=[24.7,38.4])$ and teleportation which took on average $49.7 \mathrm{~s}(\mathrm{CI}=[40.3,58.3])$. Pairwise comparisons (Figure 7-left) show that flight clearly outperformed 3D cone drag, worldin-miniature had comparable performance to 3D cone drag, and 3D cone drag outperformed teleportation.

Orientation. Participants again performed best with flight with an average of $16.3^{\circ}(\mathrm{CI}=[11.5,20.9])$ of point-back error. This was close to world-in-miniature which had an average of $17.5^{\circ}(\mathrm{CI}=[13.1,21.9])$ of error. 3D cone drag had an average of $25.3^{\circ}(\mathrm{CI}=[17.8,37.6])$ of error which outperformed teleportation 
which had an average of $32.2^{\circ}(\mathrm{CI}=[27.7,36.8])$ of error. Pairwise comparisons (Figure 7-right) show that participants were consistently more accurate when using flight than 3D cone drag.

\section{DISCUSSION}

Overall, participants found flight much easier to use than any other individual technique and preferred to use it when possible. However, participants also highlighted distinct advantages of each of the other techniques and frequently combined techniques to leverage their respective strengths.

\subsection{Participant Feedback}

Flight. Overall, participants articulated a strong preference for flight and found it very easy to use (Figure 8). In Study 1, 7 participants rated flight as their favorite while 1 rated it as least favorite. In Study 2, five participants rated flight as their favorite and 0 as least favorite. Overall, participants found flight intuitive to use and effective at all tasks. The only complaints were that it could be nauseating at higher velocities and that movement could be a bit slow. P5 and P6 noted that they felt immersed in the environment while using flight.

World-in-Miniature. Participants were more divided in their opinion on world-in-miniature and opinions varied on whether it was easy or difficult (Figure 8). This matches our study results where world-in-miniature ranked near the middle on both performance measures. In Study 1, 3 participants rated world-inminiature as their favorite and 3 as least favorite. In Study 2, 1 participant rated it as their favorite if combined with flight and 2 as least favorite. Participants liked that they could use their whole body to control the token and found it to be a fast method for gaining an overview of the scene. However, they also found that precise control was challenging and participants would often spend a lot of time struggling with fine-grained navigation.

Teleportation. Participants disliked when they were forced to use teleportation and found it difficult to use (Figure 8). This matches our study results that consistently found teleportation to perform poorly compared to other navigation techniques. In Study 1, 6 participants rated teleportation as their least favorite and 0 as favorite. In Study 2, 1 participant ranked teleportation combined with flight as their favorite and 2 participants ranked it as their least favorite. Participants felt that it was ill-suited for the tasks they were asked to perform. The main reason for this was that participants found it difficult to gain an overview of the scene. P8 and P12 expressed that teleportation — by forcing them to teleport up to high points on the mountains to survey the map for coins - felt that more like exploring a game world than the other two techniques. 
However, the instantaneous nature of teleportation for line-of-sight movements made it attractive as a supplementary technique.

3D Cone Drag. Participants responses to 3D cone drag were mixed but participants generally preferred other navigation techniques when given the choice (Figure 8). Participants' difficulty rating for the technique was surprising, since 3D cone drag performed comparably to world-in-miniature in both performance measures but participants still ranked it as much more difficult. One participant ranked it as their favorite technique and 4 as least favorite. Participants liked that $3 \mathrm{~d}$ cone drag was very precise, allowing for fine movements. Several participants found that using the technique was very fun and made them feel like Tarzan or Spiderman (P13). However, when grabbing from far away, some participants found that the velocity was too fast and could be nauseating. Two participants also found that the repeated arm motions were tedious and tiring to use for longer periods.

\subsection{Combinations}

Interestingly, when participants in our studies had access to all the techniques simultaneously, they tended to combine techniques to address the relative weaknesses of individual approaches. We observed five unique combinations of techniques across the two studies (Figure 9) and suggest some combination guidelines.

Teleportation + Flight. Five total participants combined these approaches, using teleportation for rapid, longdistance travel that might otherwise be tedious. Participants would fly directly up into the air to get a good view of the scene and their target, teleport as close to the destination as possible, then fly the remaining distance.

World-in-Miniature + Flight. Similarly, 5 participants used world-in-miniature to cover large distances, before shifting to flight for small precision movements. Participants would often move upward to get a good overview of the scene using world-in-miniature then use it to get as close as possible to their target, before shifting to flight for the final more difficult movements.

3D Cone Drag + Flight. In our second study, participants used 3D cone drag in a similar way - first moving up to gain an overview of the scene and then grabbing to move most of the way to their target before flying the last bit.
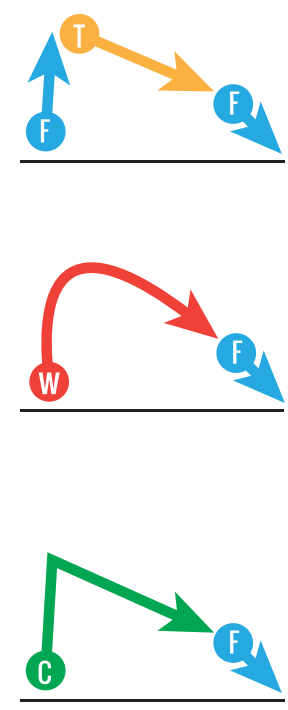
3D Cone Drag + Teleportation + Flight. One participant combined three techniques to great effect, using 3D cone drag to move up to a better vantage point, and then combining teleportation and flight to get close.

In all of these cases, participants used flight mostly for precise movements, while relying on teleportation, world-inminiature, and 3D cone drag for longer transits across the environment. Participants found world-in-miniature and 3D cone drag especially useful for moving upward quickly to obtain an overview of the scene. Even when given access to

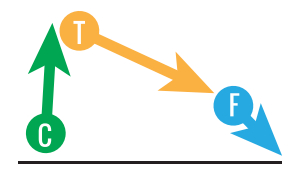
all techniques, 5 of the 14 participants still relied exclusively on flight.

Pairing Navigation Techniques Effectively. Navigation in large VR environments requires the capacity for both precise local movements and large, quick movements through the scene. Mechanisms for obtaining an overview of the environment are also particularly valuable, especially when environments include complex terrain or obstructions. Many navigation techniques fit several of these three qualifications. For example, flight can be used for high precision movement and can easily be used to obtain an overview, making it the most reliable single technique out of those we evaluated. However, when used for long, protracted movements, it (as well as the other continuous techniques) may trigger nausea or discomfort. As a result, pairing flight with discrete teleportation and/or a continuous technique like world-in-miniature or 3D cone drag allows viewers to navigate efficiently and reducing their risk of disorientation.

\section{CONCLUSION}

When working in a large immersive virtual environment every navigation technique has its own strengths and weaknesses. We have highlighted some of these challenges and discussed how combinations can counteract technique-specific weaknesses and greatly increase user power and freedom. When considering which techniques to support in a system, designers confronted with large open environments will typically want to include some variant of flight (the most versatile stand-alone technique). However, complementing flight with techniques that support more rapid movement or easier access to overviews has the potential to create a more satisfying viewer experience.

Acknowledgements. We acknowledge the support of the Natural Sciences and Engineering Research Council of Canada (NSERC) [RGPIN-2016-04564] as well as our colleagues who helped test and give feedback on the techniques and paper.

\section{References}

1. Publication Manual of the American Psychological Association. American Psychological Association, Washington, D.C., sixth edit edn. (2017) 
2. Argelaguet, F., Maignant, M.: GiAnt: Stereoscopic-compliant Multiscale Navigation in VEs. In: ACM VRST. pp. 269-277 (2016). https://doi.org/10.1145/2993369.2993391

3. Bowman, D.A., Johnson, D.B., Hodges, L.F.: Testbed Evaluation of Virtual Environment Interaction Techniques. Presence: Teleoperators and Virtual Environments 10, 75-95 (2001). https://doi.org/10.1162/105474601750182333

4. Bowman, D.A., Koller, D., Hodges, L.F.: Travel in immersive virtual environments: An evaluation of viewpoint motion control techniques. In: IEEEVR. pp. $45-52$ (1997)

5. Cumming, G.: The new statistics: Why and how. Psychological science 25(1), 7-29 (2014)

6. Jankowski, J., Hachet, M.: Advances in interaction with $3 \mathrm{D}$ environments. In: Computer Graphics Forum. vol. 34, pp. 152-190. Wiley Online Library (2015)

7. Jia Wang, Lindeman, R.W.: Comparing isometric and elastic surfboard interfaces for leaning-based travel in 3D virtual environments. In: IEEE 3DUI. pp. 31-38. IEEE (2012). https://doi.org/10.1109/3DUI.2012.6184181

8. Käser, D.P., Parker, E., Glazier, A., Podwal, M., Seegmiller, M., Wang, C.P., Karlsson, P., Ashkenazi, N., Kim, J., Le, A., Bühlmann, M., Moshier, J.: The Making of Google Earth VR. In: ACM SIGGRAPH 2017 Talks. pp. 63:1-63:2. ACM (2017). https://doi.org/10.1145/3084363.3085094

9. Kopper, R., Ni, T., Bowman, D.A., Pinho, M.: Design and Evaluation of Navigation Techniques for Multiscale Virtual Environments . In: IEEEVR. vol. 2006, pp. 175182 (2006). https://doi.org/10.1109/VR.2006.47

10. Krekhov, A., Cmentowski, S., Emmerich, K., Masuch, M., Krüger, J.: GulliVR. In: CHI PLAY. pp. 243-256. ACM Press (2018). https://doi.org/10.1145/3242671.3242704

11. Liu, J., Parekh, H., Al-Zayer, M., Folmer, E.: Increasing Walking in VR using Redirected Teleportation. In: ACM UIST. pp. 521-529 (2018). https://doi.org/10.1145/3242587.3242601

12. McCrae, J., Mordatch, I., Glueck, M., Khan, A.: Multiscale 3D navigation . In: I3D. pp. 7-14. ACM (2009). https://doi.org/10.1145/1507149.1507151

13. Montello, D.R.: Spatial Cognition and Architectural Space: Research Perspectives. In: Architectural Design. pp. 74-79 (2014). https://doi.org/10.1002/ad.1811

14. Peck, T.C., Fuchs, H., Whitton, M.C.: The Design and Evaluation of a LargeScale Real-Walking Locomotion Interface. IEEE TVCG 18(7), 1053-1067 (2012). https://doi.org/10.1109/TVCG.2011.289

15. Slater, M., Usoh, M., Steed, A.: Taking steps: the influence of a walking technique on presence in virtual reality. ACM TOCHI 2, 201-219 (1995). https://doi.org/10.1145/210079.210084

16. Stoakley, R., Conway, M.J., Pausch, R.: Virtual Reality on a WIM: Interactive Worlds in Miniature. In: ACM SIGCHI. pp. 265-272 (1995). https://doi.org/10.1145/223904.223938

17. Trindade, D.R., Raposo, A.B.: Improving 3D Navigation in Multiscale Environments Using Cubemap-based Techniques. In: ACM SAC. pp. 1215-1221 (2011). https://doi.org/10.1145/1982185.1982454

18. Ware, C., Osborne, S.: Exploration and Virtual Camera Control in Virtual Three Dimensional Environments. SIGGRAPH Computer Graphics pp. 175-183 (1990). https://doi.org/10.1145/91394.91442

19. Yan, Z., Lindeman, R.W., Dey, A.: Let your fingers do the walking: A unified approach for efficient short-, medium-, and long-distance travel in VR. In: IEEE 3DUI. pp. 27-30 (2016). https://doi.org/10.1109/3DUI.2016.7460027 